\title{
A Network Scanner Image Management Station
}

\author{
Jack W. London and Daniel E. Morton
}

Distributed computing can be applied to CT or MRI scanner image acquisition, printing, and archiving by interfacing the scanner with a computer network. Also on the network must be a computer workstation that has image management software to capture the images sent over the network by the scanner. This software must also allow the radiology staff to print and archive the images. Several benefits are realized by this network configuration. First, the scanner console is solely used for scanning patients; delays are not encountered because the console is being used for printing and archiving images. Also, the workstation printing software can be developed so that useful features not available at the scanner console can be incorporated. Finally, sophisticated archiving strategies can be implemented at the workstation. We have developed a network image management station at the Fox Chase Cancer Center using a reduced instruction set computer (RISC) workstation. Hardware and software is utilized to convert all scanner image data to the American College of Radiology-National Electrical Manufacturers Association format. All workstation software is developed using the open X-Windows standard. Digital audio tape is used for image archiving.

Copyright (1) 1992 by W.B. Saunders Company

KEY WORDS: image management, network, PACS, workstation, ACR-NEMA, X-Windows, DAT.

$\mathbf{T}$ HE Fox Chase Cancer Center (FCCC) is one of 28 comprehensive cancer centers in the United States. The Center is comprised of basic science research laboratories in the Institute for Cancer Research, and a 100-bed oncologic hospital. The hospital has one computed tomography (CT) scanner, and two magnetic resonance imaging (MRI) units.

Recent replacement of the CT and MRI scanners provided the opportunity to implement a Picture Archiving and Communications System (PACS) for the Center. The primary focus of the PACS is on the nonradiologist clinician (eg, oncologist, surgeon), and their need to view radiographic images along with other clinical data in textual or graphical form. The overall objective is to develop an integrated clinical workstation. This workstation will provide integrated computer access to all clinical data at the point of care, such as clinic examination rooms and nursing stations. ${ }^{1}$
The basic design principle is to use open (nonproprietary) standards when possible. Using open standards can decrease costs and increase the usefulness of the hardware and software. This adherence to standards has been obtained by using the UNIX operating system with reduced instruction set computers (RISC), ${ }^{2}$ which communicate via the Transmission Control Protocol/Internet Protocol (TCP/IP). All image data is converted from vendor proprietary format to the American College of Radiology-National Electrical Manufacturers Association (ACR-NEMA) standard., ${ }^{3,4}$ The open $\mathrm{X}$-Windows graphical user interface, ${ }^{5}$ developed at the Massachusetts Institute of Technology with major computer vendors, is used with $\mathrm{X}$-terminals in the clinic to present the clinical data. The nonimage clinical data is obtained from the FCCC hospital information system. ${ }^{6}$

A central component in this integrated clinical workstation project are the network image management stations that were developed to acquire the images from the $\mathrm{CT}$ and MRI scanners, and then provide the ability to print film and archive the images. Traditionally, the film printer is directly connected to and controlled by the scanner. However, at FCCC the new CT and MRI scanners are configured with network interface hardware and software, which connect via the FCCC local area network to "multi-vendor protocol converters" (MVPs). Also, there is no connection from the scanner to the film printer. Rather, both CT and MRI film printers have a hardware interface to "laser filming managers" (LFMs). The LFMs are also connected to the local area network. In addition, the RISC network image management workstations are local area network nodes. (Fig 1.)

Images from the scanner are sent to the MVP. The image data is converted to the

From Fox Chase Cancer Center, Philadelphia, PA 19111.

Address reprint requests to Jack $W$. London, PhD, Fox Chase Cancer Center, 7701 Burholme Ave, Philadelphia, PA 19111.

Copyright $\odot 1992$ by W.B. Saunders Company

0897-1889/92/0501-0004\$03.00/0 


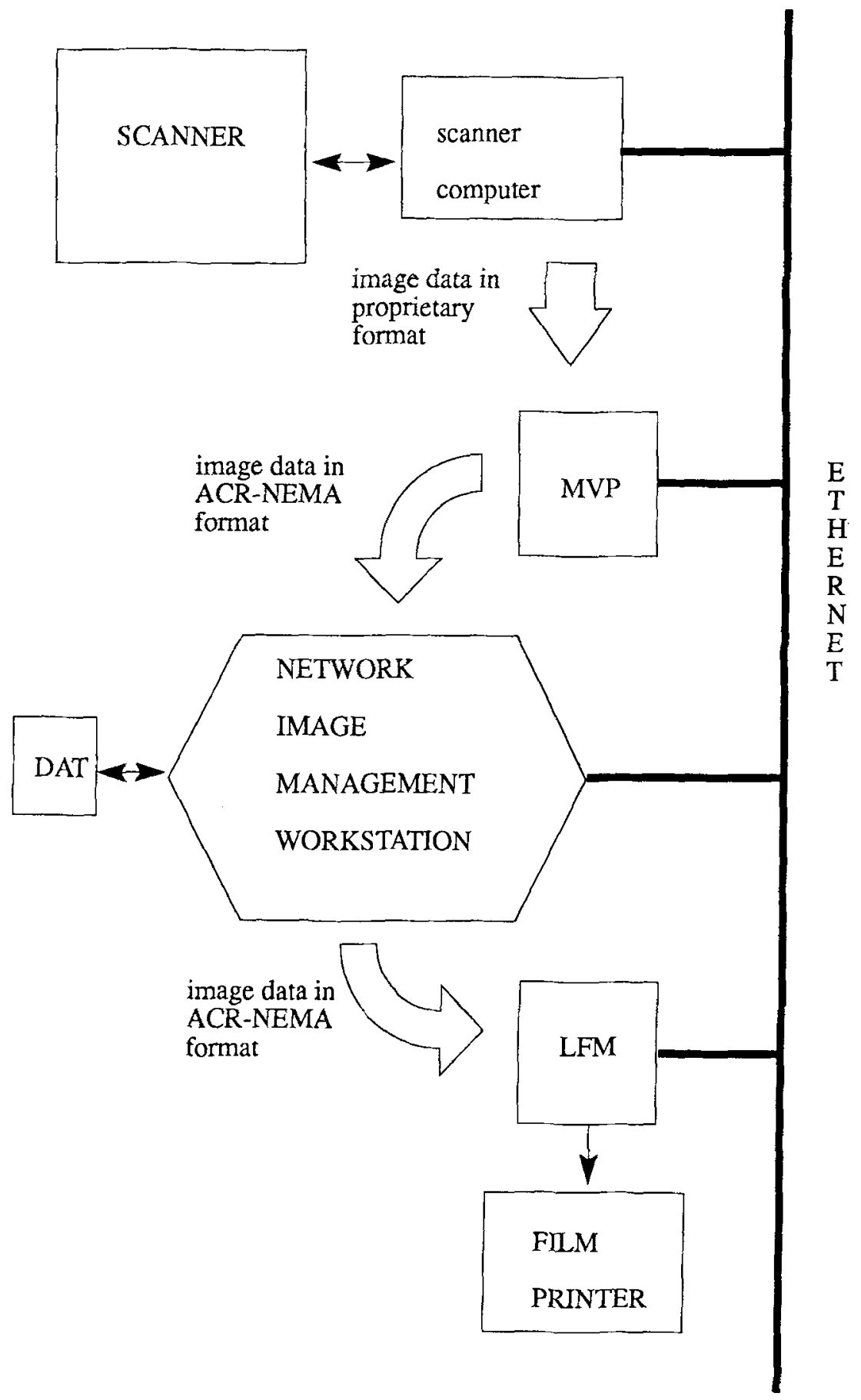

Fig 1. Configuration of the image management network at the Fox Chase Cancer Center.

ACR-NEMA format by the MVP and sent to the RISC workstation. Using workstation software developed with X-Windows, the technician can perform any needed image manipulation functions, and then print the films. Images to be printed are queued by the workstation to the LFM, which in turn sends the image data to the film printer. At the workstation, the technician also has software that allows the images to be archived to digital audio tape. (Fig 2) 

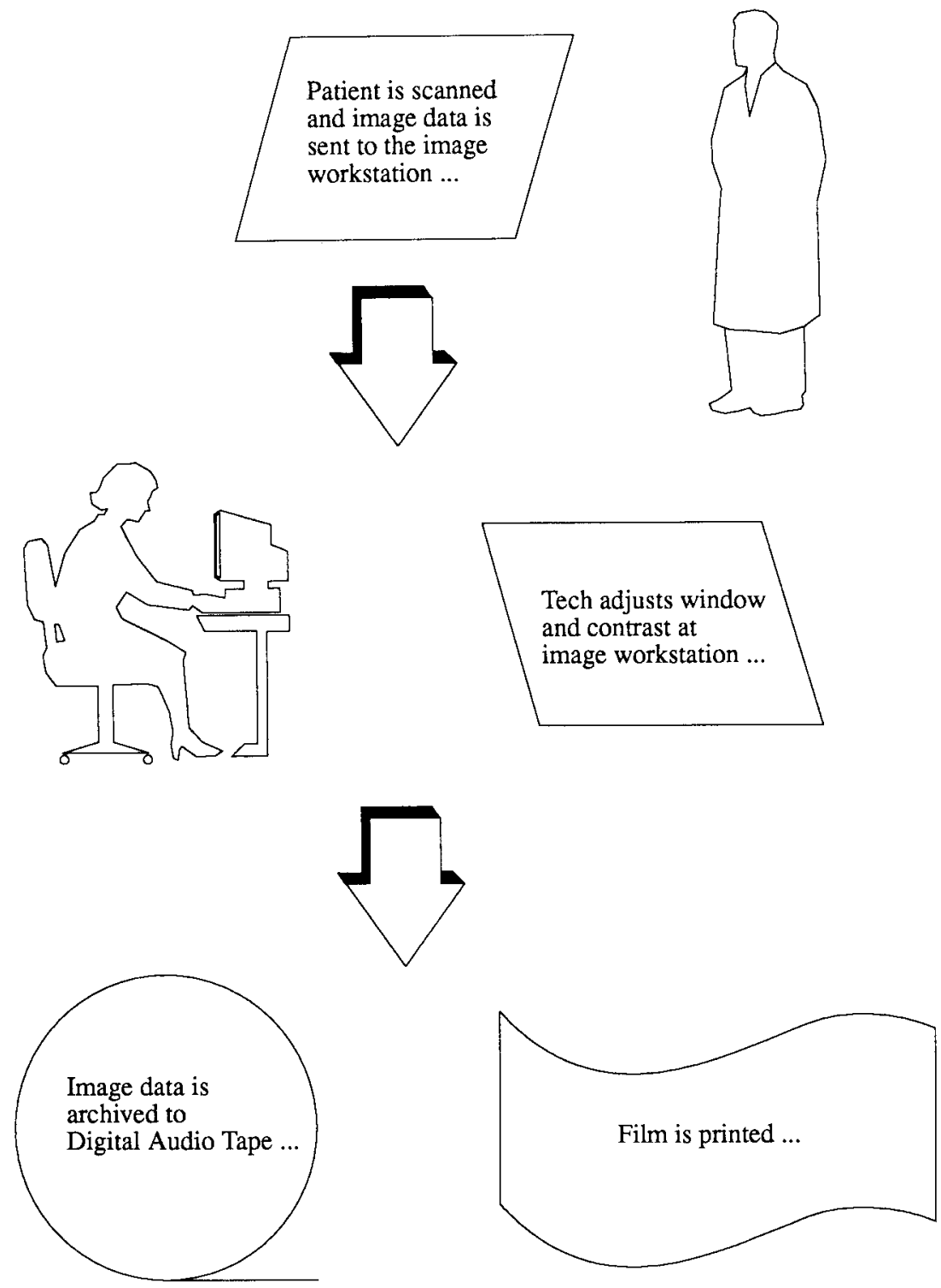

Fig 2. Diagram of work flow for images at the Fox Chase Cancer Center.

\section{SYSTEM DESCRIPTION}

The system hardware consists of scanners, scanner image format converters, computer workstations, film printer interfaces, film printers, and digital audio tape drives. The CT scanner is a Siemens (Siemens Medical Systems, Iselin, NJ) Somatom HI Q, and the MRI scanners are both Siemens Magnetom GBS IIs, one of which is primarily used for research. The RISC network image management workstations are Digital Equipment Corporation (Marlboro, MA) DECstation 5000s. Merge Technologies Inc (Milwaukee, WI) "MVP" protocol convert- ers accept image data from the scanners in Siemens proprietary format and pass them to the DECstation 5000s in the ACR-NEMA format. The DECstation 5000s pass ACR-NEMA image data to be printed to Merge Technologies "LFM" laser filming managers. The LFMs then pass the image data to Kodak Ektascan (Eastman Kodak Company, Rochester, NY) laser film printers. Archive Corporation (Costa Mesa, CA) Python 4-mm digital audio tape drives are used to backup and archive image data.

The system software includes scanner communications software, an ACR-NEMA image data 
communication and manipulation software library, and image workstation acquisition, backup, archiving, and printing software. Also a data base of information on patient images has been developed. Communication between the scanner nodes (Siemens Somatom and Magnetoms) and the Merge MVPs is over Ethernet using the DECnet protocol. Communication between the MVPs, Digital DECstation 5000s, and the Merge LFMs is over Ethernet using the TCP/IP protocol. The scanners use Siemens' software to transmit images to the Merge MVPs. Merge's "MergeCom" software library, written in $\mathrm{C}$, is used on the RISC workstations to communicate with the MVPs and LFMs. Software for the RISC workstation was developed at FCCC for use by the CT and MRI technicians for the image management functions. This software was written in $\mathrm{C}$, and uses the $\mathrm{X}$-Windows Motif toolkit (Open Software Foundation, Inc, Cambridge, MA) for the user interface. The Ingres (ASK Computer Systems, Alameda, CA) relational data base product is used for the image data base.

\section{DISCUSSION}

Image data is necessary for patient management. Point-of-care computer workstations should be able to present radiology images along with other clinical data. Because the image data will be used for review along with dictated reports, the technical specifications for the image displays are not as demanding as those for primary radiological diagnosis.

These considerations, along with a commitment to using nonproprietary standards, shaped the strategy for developing point-of-care computer workstations at FCCC.-9 Basically, the clinical workstations are X-terminals connected to a UNIX RISC compute server over Ethernet. Clinicians use the point-and-click X-Windows user interface to access patient information. This information is retrieved by the RISC compute server from hospital information system data bases residing on a VAX cluster. These data bases have patient demographics, clinical laboratory results, pathology and radiology reports, history and physicals, discharge summaries, inpatient census, and other patient data.

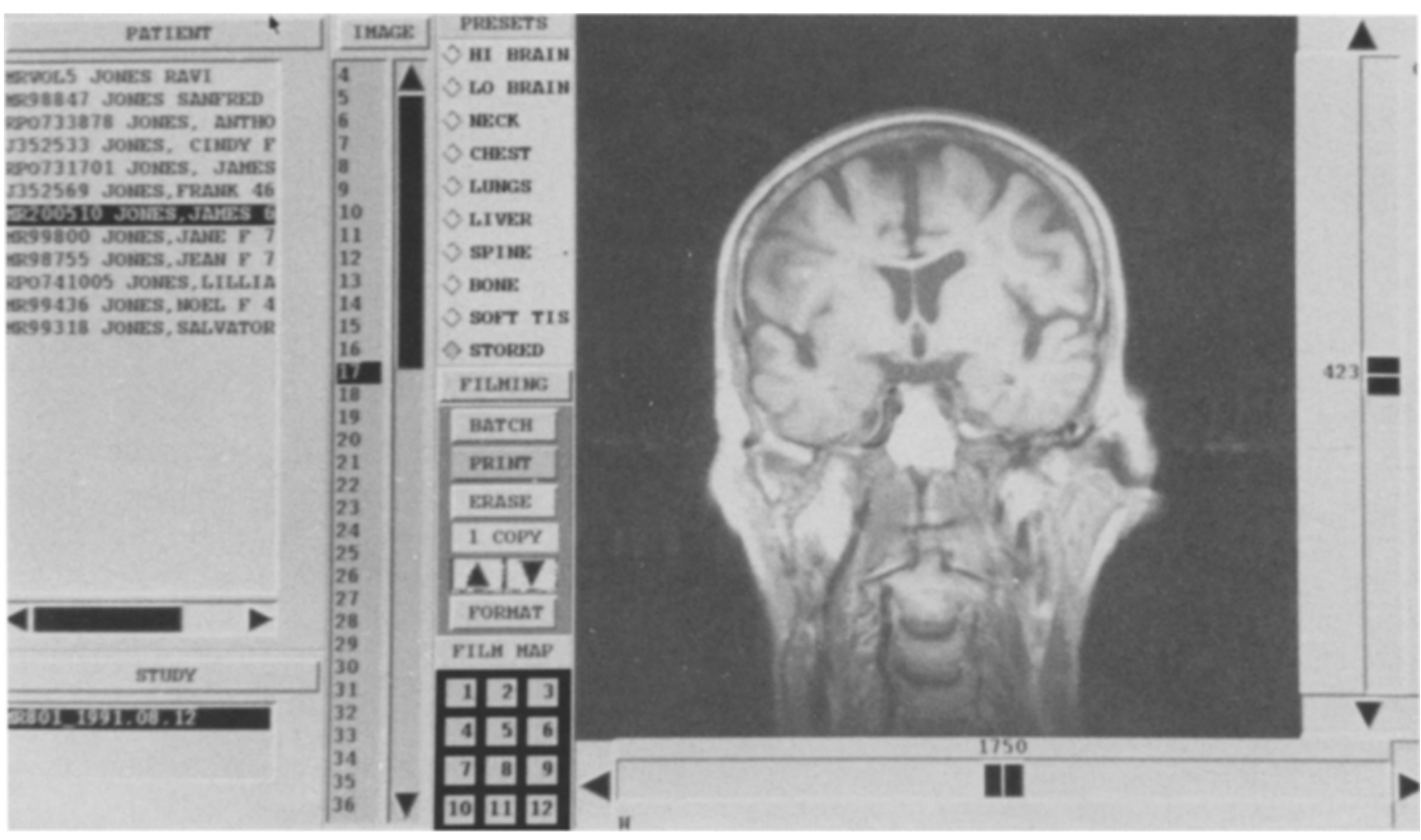

Fig 3. Workstation presentation of an MRI image. Technician presses appropriate window "buttons" to select images and send them to the film printer. Window and contrast adjustments are made by selecting desired preset levels (by pressing corresponding buttons) and/or moving arrows with a trackball. 


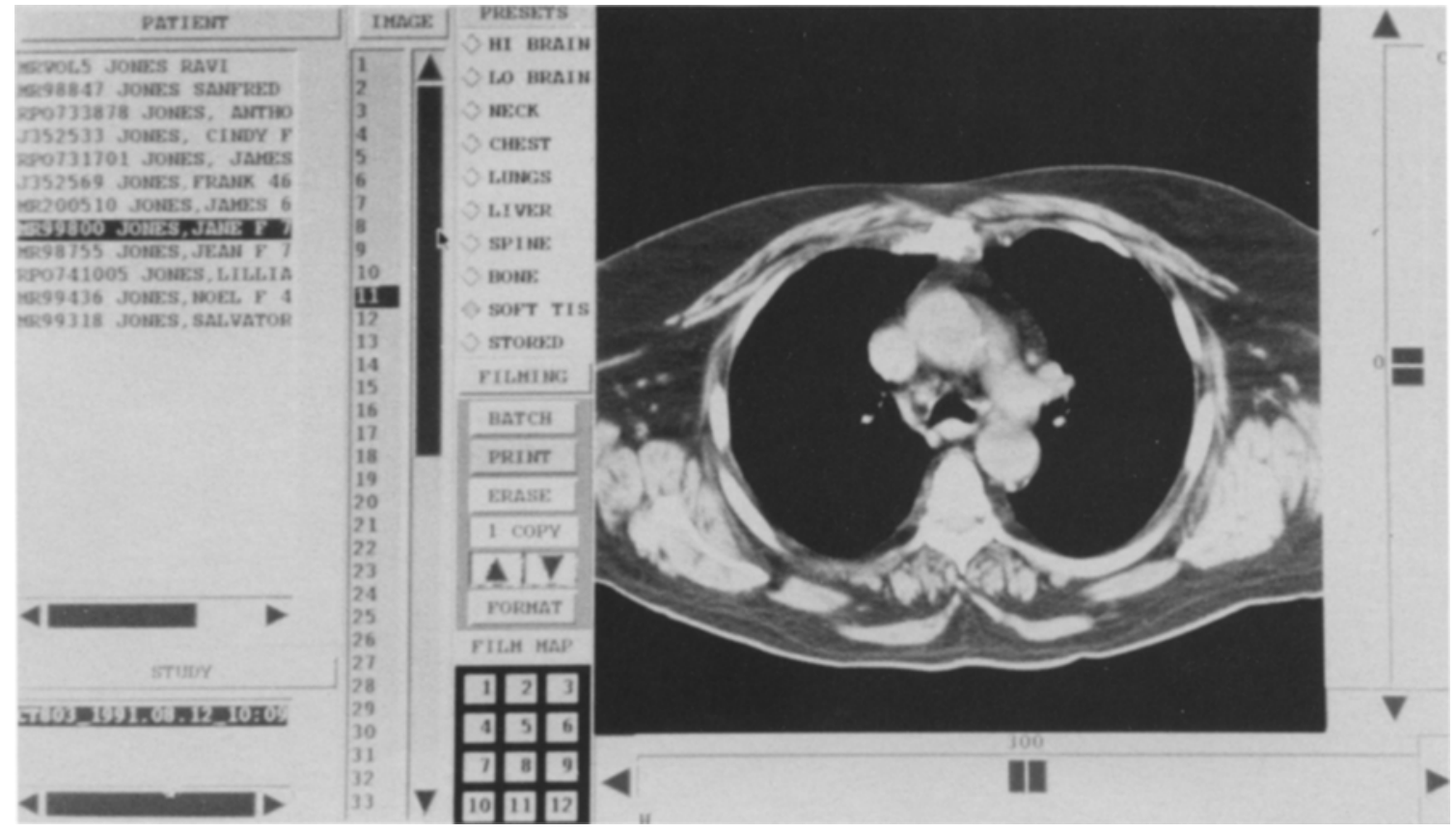

Fig 4. Workstation presentation of a CT image.

The RISC compute server also provides $\mathrm{CT}$ and MRI images acquired by the RISC image management workstations. Whereas most point-ofcare X-terminals have the capability of storing only 8-bit images, some can store 12-bits, and can thereby provide window/level functions without reloading the $\mathrm{X}$-terminal memory.

The network image management workstation is the prime prerequisite to providing images at these point-of-care X-terminals. Once the patient has been scanned the images are transmitted via the MVPs to the workstations. The scanner console is now free for the technician to commence the next patient study.

At the image management workstation the technician adjusts the window and level of the images for printing. The workstation software is designed to meet the technician's needs. For example, if image contrast is relatively constant (as is sometimes the case with CT), the contrast level can be set once for a group of images, rather than having to set the same levels for each image individually. The technician then "presses a button" on the X-Windows display, and sends the image data via the LFM to the film printer (Fig 3 and 4). At the end of each day, the day's image data are backed up to

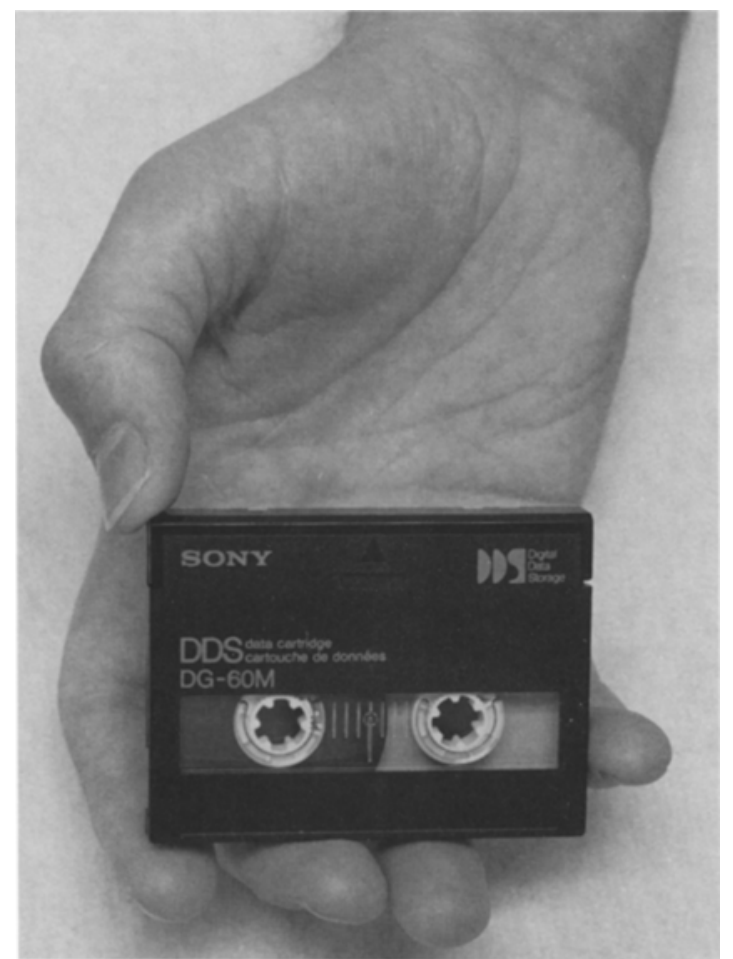

Fig 5. 4-mm digital audio tape (DAT) used for image backup and archiving. 


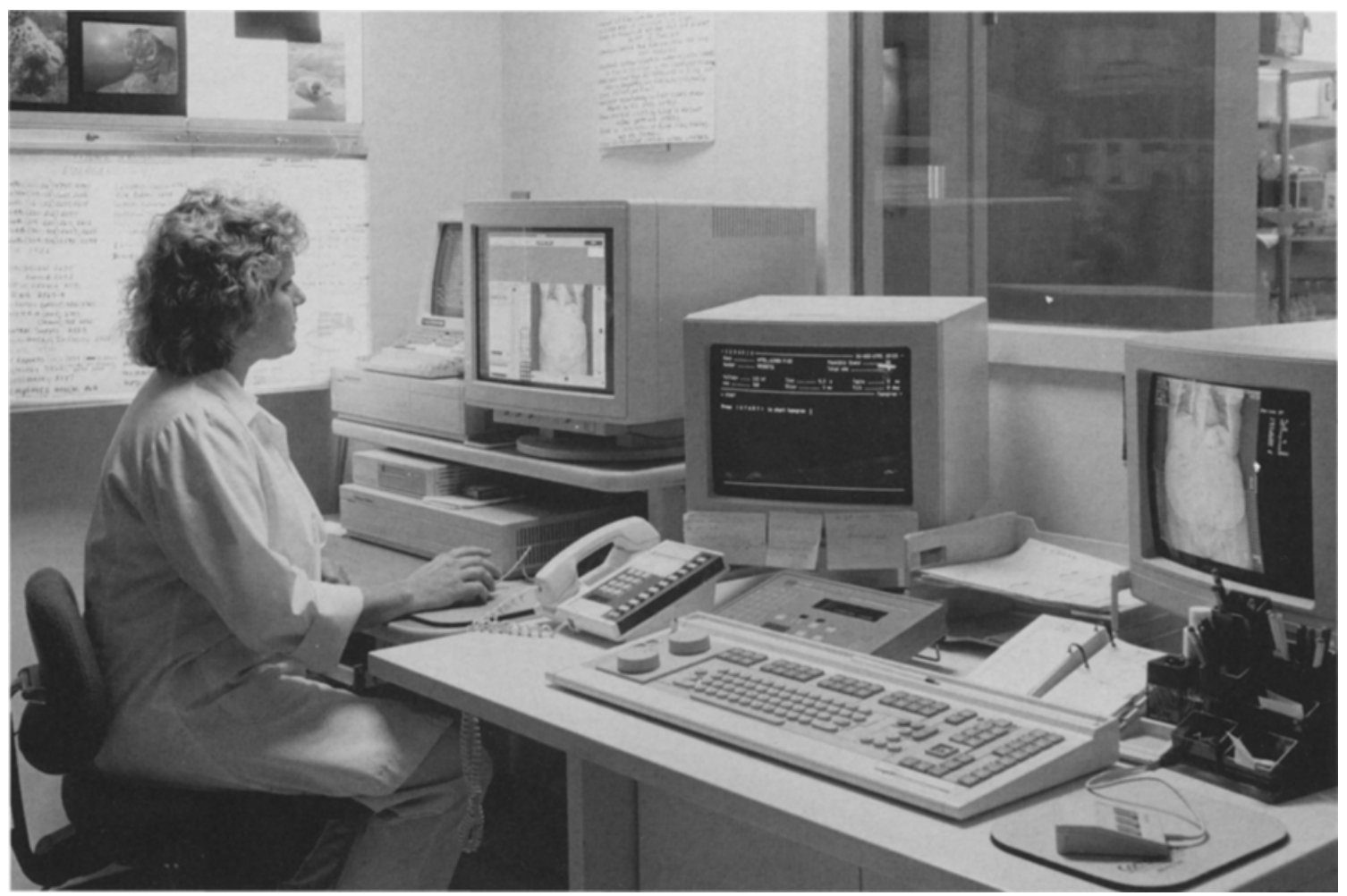

Fig 6. The CT network image management workstation is to the left of the scanner console in the CT area at the Fox Chase Cancer Center.

4-mm digital audio tape (DAT) by "pressing" another X-Window button. Digital audio tapes have capacities of at least 1.2 Gbytes (billion bytes), and can accomodate a full day on one tape.

Digital audio tapes are also being used for archiving patient data. Whereas ideally a PACS would offer on-line access to image data acquired over several years, the technology to accomplish this either does not exist or is too costly. There is also the problem that clinicians wish to retrieve image data by patient-not by date. In other words, a physician desires to retrieve all the images on various dates for a single patient, not all the images on a single date for all patients. If images are archived on chronologically-organized media (eg, optical disks created daily), the retrieval of all images for a single patient can be time-consuming and cumbersome.

Our approach is to have a single piece of media for each patient with all of their image studies. We chose 4-mm DATs because they are small (about the size of a credit card), inexpensive (currently about $\$ 11$ ), can store a life-time's worth of scan image data for an individual, and use proven magnetic tape technology (Fig 5). Furthermore, DAT drives provide very quick access to files; file access time is 20 seconds on average. The small DATs are stored in the film file room and are "pulled" each night for the next day's patients. The image data is transferred from the DAT to magnetic disk on a RISC workstation. In this manner, quick on-line access is provided to all of the patients' past study images. During the day the DATs are updated with new image data by the technicians at the workstation.

A network image management workstation has been in use with FCCC's CT scanner since April 1991 (Fig 6). Another workstation was placed in service with the MRI scanners in July 1991. Our initial experience in CT shows an increase in the number of patients scanned $(5 \%$ to $10 \%$ ) which is attributable to the greater availability of the CT console (because image 
printing and archiving are performed on the workstation). The system has been received favorably by the technicians, who benefit from the flexibility offered by the distributed network configuration and workstation software. Radiologists are pleased by the ability of the system to "customize" the film printing: because workstation software controls the film printing, the text annotation has been altered to their specifications.

A significant result of making the film printers "network devices," independent of the scanners, is that any workstation (or X-terminal) can initiate film printing. If the film printer in CT becomes inoperable, the CT films can easily be queued to the printer in MRI, and vice-versa. Furthermore, images can be sent to either film printer from any network workstation or X-terminal.

This system potentially allows for the elimination of long-term film storage. Once the primary diagnosis has been made from film, the silver could be recovered because identical films could be printed at any future time from the data archived on the patient's DAT. This film printing operation would not involve the scanner computer, and would be a straightforward transfer of image data to a RISC processor.

\section{CONCLUSION}

The network image workstation is a very "portable" device: it is a stand-alone network system that can be configured with various vendors' scanners and film printers. The workstation software operates on image data in ACR-NEMA format, and is useable insofar as proprietary image format converters are available.

This approach has inherent value in its ability to dedicate the scanner to solely scanning patients. The scanner console is no longer unavailable while being used for film printing and image archiving. Film printers become as widely and as easily accessible as networked paper printers used for word processing. Potentially, film is recycled rather than sent to long-term storage, because printing another set of identical films is readily accomplished.

Though the network image workstation configuration justifies itself, it also sets the stage for other image projects, such as the integrated point-of-care clinical workstation being developed at Fox Chase. For it is the image workstation that captures the scanner images and retrieves archived images, thus making them available for various other applications.

\section{REFERENCES}

1. London JW, Morton DE, Polekoff D, et al: The use of X-terminals as clinical workstations. J Med Sys 15:3-9, 1991

2. Patterson D: Reduced Instruction Set Computers. Communications of the ACM, 28:8-21, 1985

3. Spilker C: The ACR-NEMA Digital imaging and communications standard: A nontechnical description. $\mathrm{J}$ Digit Imag 2:127-131, 1989

4. Horii SC, Hill DG, Blume HR, et al: An update on American College of Radiology-National Electrical Manufacturers Association standards activity. J Digit Imag 3:146151,1990

5. Scheifler RW, Gettys J: The X Window system. ACM Transactions on Graphics 5:79-109, 1986

6. London JW: A cluster solution to clinical and research computing needs. Proceedings of the Ninth Annual Symposium on Computer Applications in Medical Care 722-726, 1985

7. London JW, Morton DE, Polekoff D: A Migration Path to Distributed Clinical Computing, The Fifth Annual Information Technology in the Health Sciences Conference, Memphis, TN, 1990

8. Silva JS, Zawilski AJ, O'Brian J, et al: The physician workstation: An intelligent "frontend" to a hospital information system. Proceedings of the 14th Annual Symposium on Computer Applications in Medical Care 764-767, 1990

9. Shortliffe EH, Wulfman CE, Rindfleisch TC, et al: Final report: An integrated oncology workstation. National Cancer Institute, contract NIH 263-89-C-0080, 1990 